\title{
DE-FG02-05ER15649: Transcription Factors Expressed in Lateral Organ Boundaries: Identification of Downstream Targets
}

Lateral organ initiation and patterning involve communication between the shoot apical meristem (SAM) and initiating organ primordia. The goal of this project was to characterize three boundaryexpressed genes that encode predicted transcription factors. Specifically, we have studied LATERAL ORGAN BOUNDARIES (LOB), LATERAL ORGAN FUSION1 (LOF1), and LATERAL ORGAN FUSION2 (LOF2). LOB encodes the founding member of the LOB-DOMAIN (LBD) plantspecific DNA binding transcription factor family $(1,2)$ and LOF1 and LOF2 encode paralogous MYB-domain transcription factors (3).

We characterized the genetic relationship between these three genes and other boundary and meristem genes. We also used an ectopic inducible expression system to identify direct targets of LOB.

\section{Genetic interaction studies:}

Mutations in $\angle O B$ and $\angle O F 1$ result in defects in boundary specification or maintenance, leading to fusion between axillary branches and the subtending cauline leaf. In addition, lof1 mutants lack accessory meristems (a class of axillary meristems that normally form at the base of a primary axillary branch). Mutations in lof2 do not result in a conspicuous phenotype, but enhance the lof1 mutation, such that in addition to axillary branch-leaf fusions, lof1 lof2 mutants exhibit fusions between floral pedicels and the inflorescence stem (3). While the lob and lof1 mutant phenotypes are quite specific, they are limited to only a subset of the regions expressing these genes. Both genes are part of large gene families and a number of other transcription factors are also expressed in the boundary (reviewed in 4). Thus, we hypothesize that genetic redundancy masks phenotypes in many places where these genes are expressed. The enhanced lof1 lof 2 double mutant phenotype is consistent with this hypothesis.

To understand the relationship between $\angle O B, L O F 1, \angle O F 2$, and other genes important for boundary and meristem regulation, we generated and analyzed a number of double mutant combinations between lob, lof1, lof2 and genes that function in boundary and meristem establishment or maintenance. Prior to the start of this project, we had observed that double mutants between lateral suppressor (las) and either lof1 or lof2 exhibited dramatically enhanced fusions. We had also observed that ectopic expression of the meristem-specific transcription factor SHOOT MERISTEMLESS (STM) resulted in ectopic expression of LOF1 and LOF2, suggesting that STM positively regulates LOF1/LOF2 expression. In contrast, LOF1 and LOF2 expression was expanded in the pennywise (pny) mutant, suggesting that PNY contributes to negatively regulating their expression. We had also found that the lof1 mutant suppresses some aspects of the pny single mutant and the pny poundfoolish (pnf) double mutant. We also had data implicating $\mathrm{LOB}$ as a negative regulator of response to the phytohormone brassinosteroid (BR), based on $L O B$ ectopic expression. In this project, we explored interactions and our genetic interaction data are summarized in Table 1.

Our data indicate that $\angle O B$ and $L O F 1 / L O F 2$ define two parallel pathways to control boundary specification or elaboration. In addition, the enhanced meristem defects revealed when lof1 or lof2 were combined with stm-10 (a weak allele), las, cuc2, and cuc3 indicate that LOF1 and LOF2 contribute to maintenance of the shoot apical meristem and axillary meristems.

II. Identification of LOB targets.

We used an ectopic inducible expression system that is based on the steroid-inducible glucocorticoid receptor (GR) to identify LOB targets. A transcription factor (TF) that is fused to the 
hormone-binding domain of GR is prevented from entering the nucleus in the absence of steroid hormone. Upon application of a steroid hormone such as dexamethasone (DEX), the TF-GR fusion protein is transported to the nucleus to regulate the expression of downstream genes. We generated transgenic Arabidopsis plants that express LOB fused to GR, under control of the ubiquitously expressed CaMV 35S promoter. To identify downstream targets of LOB, we conducted Affymetrix gene chip experiments with $L O B-G R$ plants, following a short period of exposure to DEX. We treated 7-day-old wild-type Columbia and 35S:LOB-GR seedlings with either mock, DEX, cycloheximide (CHX), or DEX + CHX. Three biological replicates were performed. RNAs were isolated and used in hybridization experiments to the Affymetrix Ath1 chip. These array experiments identified 113 genes whose transcript levels increased or decreased by 2-fold or more $(\mathrm{P} \leq 0.01)$ in DEX- and DEX + CHX-treated $L O B-G R$ plants and were unchanged in wild-type (Table 2). These genes are putative direct targets of LOB.

Approximately $50 \%$ of the putative LOB targets are predicted to encode enzymes involved in cell wall modification such as expansins, glycosyl transferases, pectin methyl transferases, hydrolases, and glycosylases. These data indicate that LOB plays a major role in sculpting the composition of the cell wall in the boundary. We also identified a number of genes that are predicted to function in different hormone signaling pathways, including BR, auxin, cytokinin, gibberellin and ethylene, suggesting that LOB may function to integrate multiple hormone responses in the organ boundary

\section{$\angle O B$ regulates $B R$ accumulation}

Given our previous data suggesting that $\angle O B$ is a negative regulator of BR responses, we focused our attention on two putative targets that are implicated in BR signaling: BAS1 and BRL3. $B A S 1$ encodes a cytochrome P450 enzyme that hydroxylates active BRs, resulting in their inactivation (5), while BRL3 encodes a leucine-rich-repeat receptor kinase that is one of a family of $\mathrm{BR}$ receptors (6). Using independent biological replicates and an induction time course, we confirmed that the transcript levels of both genes increase within 30 minutes of LOB-GR activation by DEX, and this positive regulation occurs in the presence of the translation inhibitor cycloheximide, indicating that they are likely to be directly regulated by LOB. Furthermore, two copies of the LOB binding site, in inverted orientation and separated by a few nucleotides, are present in the BAS1 upstream regulatory region. Using EMSAs, we demonstrated that LOB binds to the BAS1 promoter in vitro, but does not bind when either LOB-binding site is mutated. To examine LOB association with the BAS1 promoter in vivo, we used a LOB antibody in chromatin immunoprecipitation (ChIP) experiments with DEX- and mock-treated 35S:LOB-GR seedlings. In DEX-treated samples we detected enrichment of a $\sim 200 \mathrm{bp}$ fragment spanning the LOB binding sites in the BAS1 promoter. These data suggest that LOB directly activates BAS1 to inactivate BRs. Reduced BR signaling is likely necessary for boundary cell specification, as BRs promote cell division and expansion (7) and boundary cells divide slowly and are relatively unexpanded $(3,8)$.

Assuming BAS1 regulation is a normal function of $L O B$, we hypothesized that reduced BAS1 expression contributed to the fusion phenotype in the lob mutant. To test this, we expressed BAS1 under control of the $\angle O B$ promoter in wild-type and lob-3 mutant plants. In a wild-type background, $p L O B: B A S 1$ plants were morphologically normal. In lob mutants, $p L O B: B A S 1$ expression rescued the fusion defect (5 independent single-locus transgenics were analyzed), which could be quantified by measuring the region of contact between the axillary stem and cauline leaf. Thus, our data indicate that expression of BAS1 in the domain of $L O B$ expression is sufficient to rescue the lob mutant phenotype.

A target of LOB transcriptional regulation should have expression overlapping that of $L O B$. To examine the BAS1 expression pattern, we generated a pBAS1:BAS1-GUS construct containing 6.4 $\mathrm{kb}$ of upstream BAS1 sequence. In general, GUS activity in $p B A S 1: B A S 1-G U S$ transgenic plants was observed in regions undergoing active growth, such as young leaves, floral buds, and lateral root primordia. GUS expression was generally broader than that of $L O B$, but showed apparent 
overlap at the base of leaves, base of floral organs, and in paraclade junctions. Mutation of LOBbinding sites in the pBAS1:BAS1-GUS construct did not significantly alter expression however, suggesting that other regulatory sites also contribute to BAS1 expression.

Although BAS1 can suppress the lob mutant phenotype, bas 1 mutants do not exhibit fusion defects. It is likely that the lob mutant phenotype results from alteration in the regulation of multiple genes. Consistent with this idea, the bas 1 mutant does not appreciably suppress the phenotypes of 35S:LOB-GR plants, whereas bzr1-d, which produces a stabilized BR-responsive transcription factor (9), partially suppresses the $L O B-G R$ phenotype.

To test the hypothesis that boundary specification or integrity requires limited BR signaling, we expressed DWF4, which encodes a rate-limiting step in BR biosynthesis $(10,11)$, under control of the $L O B$ promoter to increase BR levels. pLOB:DWF4 plants exhibited fusion phenotypes similar to the lob mutant, providing confirmation that BR accumulation or response must be limited in the organ boundary.

Our data indicate that LOB acts as a negative regulator of BR signaling by increasing BAS1 levels, which results in a reduction in the availability of active BRs. $\angle O B$ expression is also positively regulated by $B R s$, indicating the presence of a feedback loop involving BR regulation of LOB accumulation and LOB repression of BR accumulation. More puzzling is the positive regulation of $B R L 3$, which is presumed to increase sensitivity to BRs. These data seem to indicate that LOB functions to fine-tune BR responses by altering both the levels of active BR and the response. The work demonstrates the developmental importance of regulating BR responses and indicates that formation of a BR minimum in organ boundaries is critical for boundary integrity.

\section{Publications resulting from project}

Lee, D. K., Geisler, M. and Springer, P. S. (2009). LATERAL ORGAN FUSION1 and LATERAL ORGAN FUSION2 function in lateral organ separation and axillary meristem formation in Arabidopsis. Development 136, 2423-2432.

Bell, E. M., Lin, W.-C., Husbands, A., Yu, L., Jaganatha, V., Jablonska, B., Mangeon, A., Neff, M., Girke, T. and Springer, P. S. (2010). Arabidopsis LATERAL ORGAN BOUNDARIES negatively regulates brassinosteroids to limit growth in organ boundaries. In preparation.

Lee, D. K., Wu, S., Smith, H. M. S., and Springer, P. S. (2010) The MYB-transcription factor LOF1 functions downstream of STM in meristem maintenance and boundary specification. In preparation. 


\section{References Cited}

1. Husbands, A., Bell, E.M., Shuai, B., Smith, H.M., and Springer, P.S. (2007). LATERAL ORGAN BOUNDARIES defines a new family of DNA-binding transcription factors and can interact with specific bHLH proteins. Nucleic Acids Res. 35, 6663-6671.

2. Shuai, B., Reynaga-Peña, C.G., and Springer, P.S. (2002). The LATERAL ORGAN BOUNDARIES gene defines a novel, plant-specific gene family. Plant Physiol. 129, 747-761.

3. Lee, D.K., Geisler, M., and Springer, P.S. (2009). LATERAL ORGAN FUSION1 and LATERAL ORGAN FUSION2 function in lateral organ separation and axillary meristem formation in Arabidopsis. Development 136, 2423-2432.

4. Rast, M., and Simon, R. (2008). The meristem-to-organ boundary: more than an extremity of anything. Curr. Opin. Genet. Dev. 18, 287-294.

5. Neff, M.M., Nguyen, S.M., Malancharuvil, E.J., Fujioka, S., Noguchi, T., Seto, H., Tsubuki, M., Honda, T., Takatsuto, S., Yoshida, S., et al. (1999). BAS1: A gene regulating brassinosteroid levels and light responsiveness in Arabidopsis. Proc. Natl. Acad. Sci. USA 96, 15316-15323.

6. Caño-Delgado, A., Yin, Y., Yu, C., Vafeados, D., Mora-Garcia, S., Cheng, J.C., Nam, K.Y., Li, J., and Chory, J. (2004). BRL1 and BRL3 are novel brassinosteroid receptors that function in vascular differentiation in Arabidopsis. Development 131, 5341-5351.

7. Friedrichsen, D., and Chory, J. (2001). Steroid signaling in plants: from the cell surface to the nucleus. Bioessays 23, 1028-1036.

8. Hussey, G. (1971). Cell division and expansion and resultant tissue tensions in shoot apex during formation of a leaf primordium in tomato. J. Exp. Bot. 22, 702-714.

9. He, J.X., Gendron, J.M., Sun, Y., Gampala, S.S., Gendron, N., Sun, C.Q., and Wang, Z.Y. (2005). BZR1 is a transcriptional repressor with dual roles in brassinosteroid homeostasis and growth responses. Science 307, 1634-1638.

10. Choe, S., Dilkes, B.P., Fujioka, S., Takatsuto, S., Sakurai, A., and Feldmann, K.A. (1998). The DWF4 gene of Arabidopsis encodes a cytochrome P450 that mediates multiple 22alphahydroxylation steps in brassinosteroid biosynthesis. Plant Cell 10, 231-243.

11. Kim, H.B., Kwon, M., Ryu, H., Fujioka, S., Takatsuto, S., Yoshida, S., An, C.S., Lee, I., Hwang, I., and Choe, S. (2006). The regulation of DWARF4 expression is likely a critical mechanism in maintaining the homeostasis of bioactive brassinosteroids in Arabidopsis. Plant Physiol. 140, 548-557.

12. Aida, M., Ishida, T., Fukaki, H., Fujisawa, H., and Tasaka, M. (1997). Genes involved in organ separation in Arabidopsis: an analysis of the cup-shaped cotyledon mutant. Plant Cell 9, 841857.

13. Vroemen, C.W., Mordhorst, A.P., Albrecht, C., Kwaaitaal, M.A., and de Vries, S.C. (2003). The CUP-SHAPED COTYLEDON3 gene is required for boundary and shoot meristem formation in Arabidopsis. Plant Cell 15, 1563-1577.

14. Greb, T., Clarenz, O., Schafer, E., Muller, D., Herrero, R., Schmitz, G., and Theres, K. (2003). Molecular analysis of the LATERAL SUPPRESSOR gene in Arabidopsis reveals a conserved control mechanism for axillary meristem formation. Genes Dev. 17, 1175-1187.

15. Keller, T., Abbott, J., Moritz, T., and Doerner, P. (2006). Arabidopsis REGULATOR OF AXILLARY MERISTEMS1 controls a leaf axil stem cell niche and modulates vegetative development. Plant Cell 18, 598-611.

16. Müller, D., Schmitz, G., and Theres, K. (2006). Blind homologous R2R3 Myb genes control the pattern of lateral meristem initiation in Arabidopsis. Plant Cell 18, 586-597. 
17. Ha, C.M., Jun, J.H., Nam, H.G., and Fletcher, J.C. (2004). BLADE-ON-PETIOLE1 encodes a $\mathrm{BTB} / \mathrm{POZ}$ domain protein required for leaf morphogenesis in Arabidopsis thaliana. Plant Cell Physiol. 45, 1361-1370.

18. Long, J.A., and Barton, M.K. (1998). The development of apical embryonic pattern in Arabidopsis. Development 125, 3027-3035.

19. Douglas, S.J., Chuck, G., Dengler, R.E., Pelecanda, L., and Riggs, C.D. (2002). KNAT1 and ERECTA regulate inflorescence architecture in Arabidopsis. Plant Cell 14, 547-558.

20. Smith, H.M., Campbell, B.C., and Hake, S. (2004). Competence to respond to floral inductive signals requires the homeobox genes PENNYWISE and POUND-FOOLISH. Curr. Biol. 14, 812-817. 
Table 1. Genetic interactions observed in this project.

$\$$ interactions that were observed prior to the start of this project.

\begin{tabular}{|c|c|c|c|}
\hline & $\angle O B$ & LOF1 & LOF2 \\
\hline \multicolumn{4}{|l|}{ Boundary genes } \\
\hline$L O B$ & -- & Additive $^{\$}$ & Additive $^{\$}$ \\
\hline LOF1 & Additive $^{\$}$ & -- & Enhanced/additional fusions ${ }^{\$}$ \\
\hline LOF2 & Additive $^{\$}$ & Enhanced/additional fusions $^{\$}$ & -- \\
\hline $\begin{array}{l}\text { CUP-SHAPED } \\
\text { COTYLEDON1 (CUC1) } \\
\text { (12) }\end{array}$ & Additive & Additive & Additive \\
\hline $\begin{array}{l}\text { CUP-SHAPED } \\
\text { COTYLEDON2 (CUC2) } \\
\text { (12) }\end{array}$ & Additive & $\begin{array}{l}\text { Enhanced/additional fusions } \\
\text { and meristem defects }\end{array}$ & $\begin{array}{l}\text { Enhanced/additional fusions } \\
\text { and meristem defects }\end{array}$ \\
\hline $\begin{array}{l}\text { CUP-SHAPED } \\
\text { COTYLEDON3 (CUC3) } \\
\text { (13) }\end{array}$ & Additive & $\begin{array}{l}\text { Enhanced/additional fusions } \\
\text { and meristem defects }\end{array}$ & $\begin{array}{l}\text { Enhanced/additional fusions } \\
\text { and meristem defects }\end{array}$ \\
\hline $\begin{array}{l}\text { LATERAL } \\
\text { SUPPRESSOR (LAS) } \\
(14)\end{array}$ & $\begin{array}{l}\text { Enhanced } \\
\text { fusion }\end{array}$ & $\begin{array}{l}\text { Enhanced/additional fusions } \\
\text { and meristem defects }\end{array}$ & $\begin{array}{l}\text { Enhanced/additional fusions } \\
\text { and meristem defects } \$\end{array}$ \\
\hline $\begin{array}{l}\text { REGULATORS OF } \\
\text { AXILLARY } \\
\text { MERISTEMS (RAX) } \\
(15,16)\end{array}$ & Additive & Additive & Additive \\
\hline $\begin{array}{l}\text { BLADE ON PETIOLE1 } \\
\text { (BOP1) (17) }\end{array}$ & Additive & Additive & Additive \\
\hline \multicolumn{4}{|l|}{ Meristem genes } \\
\hline $\begin{array}{l}\text { SHOOT } \\
\text { MERISTEMLESS } \\
\text { (STM) (18) }\end{array}$ & Additive & $\begin{array}{l}\text { Meristem defect in weak stm- } \\
10 \text { enhanced }\end{array}$ & $\begin{array}{l}\text { Meristem defect in weak stm- } \\
10 \text { enhanced }\end{array}$ \\
\hline $\begin{array}{l}\text { BREVIPEDICELLUS } \\
(B P)(19)\end{array}$ & Additive & Additive & Additive \\
\hline PENNYWISE (PNY) & Additive & pny phenotype suppressed & pny phenotype suppressed \\
\hline $\begin{array}{l}\text { POUNDFOOLISH } \\
\text { (PNF) (20) }\end{array}$ & Additive & $\begin{array}{l}\text { No interaction with pnf, but } \\
\text { pny pnf double mutant } \\
\text { phenotype suppressed }\end{array}$ & $\begin{array}{l}\text { No interaction with pnf, but } \\
\text { pny pnf double mutant } \\
\text { phenotype suppressed }\end{array}$ \\
\hline
\end{tabular}


Table 2: Genes differentially expressed in DEX treated 35S:LOB-GR seedlings compared to mock treated and CHX/DEX treated compared to $\mathrm{CHX}^{1}$

1 Differentially expressed genes in LOB-GR DEX-treated (D) compared to mock

${ }^{2}$ Arabidopsis Genome Initiative identifier

${ }^{3} \log 2$ Fold Change, means of three biological replicates. $\log 2 \mathrm{FC} \geq 1$ or $\leq-1$ in LOB-GR

${ }^{4}$ False discovery rate (FDR) adjusted $p$-value. $P \leq 0.001$ in LOB-GR D/M

${ }^{5}$ Multiple LOB binding sites present in promoter

\begin{tabular}{|c|c|c|c|c|c|c|}
\hline & & & & & \multirow[b]{2}{*}{$\begin{array}{l}\text { Multiple } \\
\text { Binding } \\
\text { sites }^{5}\end{array}$} \\
\hline $\mathrm{AGI}^{2}$ & Description & $\begin{array}{c}\log 2 F C \\
\text { LOB-GR } \\
\text { CD/C }\end{array}$ & $\begin{array}{c}\text { p-value } \\
\text { LOB-GR } \\
\text { CD/C } \\
\end{array}$ & $\begin{array}{c}\log 2 F C \\
\text { LOB-GR } \\
D / M^{3}\end{array}$ & $\begin{array}{c}\text { p-value } \\
\text { LOB-GR } \\
\text { D/M }\end{array}$ & \\
\hline AT3G13380 & $\begin{array}{l}\text { leucine-rich repeat family protein / protein kinase family protein, } \\
\text { contains Pfam domains PF00560: Leucine Rich Repeat and PF00069: } \\
\text { Protein kinase domain }\end{array}$ & 2.101 & $4.2 \mathrm{E}-12$ & 3.242 & 1.1E-14 & yes \\
\hline AT2G26710 & $\begin{array}{l}\text { Encodes a member of the cytochrome p450 family. Involved in } \\
\text { brassinolide metabolism. Mediates response to a variety of light signals } \\
\text { including hypocotyl elongation and cotyledon expansion. }\end{array}$ & 4.938 & $6.4 \mathrm{E}-15$ & 3.216 & $1.4 \mathrm{E}-10$ & yes \\
\hline AT5G66460 & $\begin{array}{l}\text { (1-4)-beta-mannan endohydrolase, putative, similar to (1-4)-beta- } \\
\text { mannan endohydrolase (Coffea arabica) GI:10178872; contains Pfam } \\
\text { profile PF00150: Cellulase (glycosyl hydrolase family 5) }\end{array}$ & 4.186 & $2.2 \mathrm{E}-16$ & 3.180 & $5.5 \mathrm{E}-14$ & \\
\hline AT3G22840 & $\begin{array}{l}\text { chlorophyll A-B binding family protein / early light-induced protein } \\
\text { (ELIP), identical to early light-induced protein; ELIP (Arabidopsis } \\
\text { thaliana) GI:1872544; contains Pfam profile: PF00504 chlorophyll A-B } \\
\text { binding protein; identical to cDNA early light-i }\end{array}$ & 2.660 & 7.6E-12 & 2.219 & 1.1E-09 & yes \\
\hline AT5G57785 & expressed protein & 1.775 & $5.8 \mathrm{E}-07$ & 2.177 & 7.7E-08 & \\
\hline AT3G51660 & $\begin{array}{l}\text { macrophage migration inhibitory factor family protein / MIF family } \\
\text { protein, contains Pfam profile: PF01187 Macrophage migration inhibitory } \\
\text { factor family(MIF) }\end{array}$ & 3.946 & $6.4 \mathrm{E}-15$ & 1.898 & $1.3 \mathrm{E}-08$ & \\
\hline AT1G64405 & expressed protein & 2.727 & $3.9 \mathrm{E}-12$ & 1.858 & $1.4 \mathrm{E}-08$ & \\
\hline AT5G01600 & $\begin{array}{l}\text { ferritin } 1 \text { (FER1), identical to ferritin (Arabidopsis thaliana) GI:1246401, } \\
\text { GI:8163920 }\end{array}$ & 1.421 & $2.8 \mathrm{E}-04$ & 1.839 & 2.8E-05 & \\
\hline AT5G02260 & $\begin{array}{l}\text { expansin, putative (EXP9), similar to expansin precursor } \mathrm{Gl}: 4138914 \\
\text { from (Lycopersicon esculentum); alpha-expansin gene family, } \\
\text { PMID:11641069 }\end{array}$ & 1.223 & 1.3E-07 & 1.832 & $8.4 \mathrm{E}-10$ & \\
\hline AT3G13672 & $\begin{array}{l}\text { seven in absentia (SINA) family protein, low similarity to SP:P21461 } \\
\text { Developmental protein seven in absentia \{Drosophila melanogaster\}; } \\
\text { contains Pfam profile PF03145: Seven in absentia protein family }\end{array}$ & 2.879 & $5.2 \mathrm{E}-11$ & 1.828 & $3.1 \mathrm{E}-07$ & \\
\hline AT1G48330 & $\begin{array}{l}\text { expressed protein, similar to hypothetical protein } \mathrm{Gl}: 9294146 \text { from } \\
\text { (Arabidopsis thaliana) }\end{array}$ & 1.075 & $1.4 \mathrm{E}-04$ & 1.761 & 4.3E-07 & \\
\hline AT1G71030 & $\begin{array}{l}\text { Encodes a putative myb family transcription factor. In contrast to most } \\
\text { other myb-like proteins its myb domain consists of a single repeat. A } \\
\text { proline-rich region potentially involved in transactivation is found in the C- } \\
\text { terminal part of the protein. }\end{array}$ & 2.159 & 1.6E-07 & 1.758 & 7.0E-06 & \\
\hline AT4G28270 & $\begin{array}{l}\text { zinc finger (C3HC4-type RING finger) family protein, contains Pfam } \\
\text { profile: PF00097 zinc finger, C3HC4 type (RING finger) }\end{array}$ & 1.129 & $1.8 \mathrm{E}-06$ & 1.682 & $1.4 \mathrm{E}-08$ & \\
\hline AT4G30410 & $\begin{array}{l}\text { expressed protein, similar to cDNA bHLH transcription factor (bHLH eta } \\
\text { gene) gi: } 32563007\end{array}$ & 4.023 & $2.2 \mathrm{E}-14$ & 1.633 & $3.5 \mathrm{E}-07$ & \\
\hline AT3G18280 & $\begin{array}{l}\text { protease inhibitor/seed storage/lipid transfer protein (LTP) family } \\
\text { protein, similar to TED4 (Zinnia elegans) Gl:493721; contains Pfam } \\
\text { protease inhibitor/seed storage/LTP family domain PF00234 }\end{array}$ & 1.677 & $1.4 \mathrm{E}-11$ & 1.499 & $7.8 \mathrm{E}-10$ & \\
\hline AT1G74670 & $\begin{array}{l}\text { gibberellin-responsive protein, putative, similar to SP:P46690 } \\
\text { Gibberellin-regulated protein } 4 \text { precursor }\{\text { Arabidopsis thaliana\} GASA4; } \\
\text { contains Pfam profile PF02704: Gibberellin regulated protein }\end{array}$ & 1.132 & 9.7E-04 & 1.478 & $1.2 \mathrm{E}-04$ & \\
\hline AT2G39700 & $\begin{array}{l}\text { expansin, putative (EXP4), similar to alpha-expansin } 6 \text { precursor } \\
\text { GI:16923359 from (Cucumis sativus); alpha-expansin gene family, } \\
\text { PMID:11641069 }\end{array}$ & 2.981 & $1.6 \mathrm{E}-13$ & 1.394 & 2.7E-07 & yes \\
\hline AT4G37295 & expressed protein & 1.751 & $5.0 \mathrm{E}-10$ & 1.370 & $1.0 \mathrm{E}-07$ & \\
\hline AT3G22060 & $\begin{array}{l}\text { contains Pfam profile: PF01657 Domain of unknown function that is } \\
\text { usually associated with protein kinase domain Pfam:PF00069, however } \\
\text { this protein does not have the protein kinase domain }\end{array}$ & 1.584 & 6.7E-07 & 1.361 & $1.2 \mathrm{E}-05$ & \\
\hline AT4G24780 & $\begin{array}{l}\text { pectate lyase family protein, similar to pectate lyase GP:14289169 from } \\
\text { (Salix gilgiana) }\end{array}$ & 2.876 & $1.0 \mathrm{E}-12$ & 1.352 & 1.0E-06 & \\
\hline AT4G11140 & $\begin{array}{l}\text { encodes a member of the ERF (ethylene response factor) subfamily B-5 } \\
\text { of ERF/AP2 transcription factor family. The protein contains one AP2 } \\
\text { domain. There are } 7 \text { members in this subfamily. }\end{array}$ & 3.610 & $3.2 \mathrm{E}-15$ & 1.326 & $3.2 \mathrm{E}-07$ & yes \\
\hline AT3G61160 & $\begin{array}{l}\text { shaggy-related protein kinase beta / ASK-beta (ASK2), identical to } \\
\text { shaggy-related protein kinase beta SP:O23145 GI:2569931 from } \\
\text { (Arabidopsis thaliana) }\end{array}$ & 1.395 & $1.0 \mathrm{E}-06$ & 1.307 & $5.5 \mathrm{E}-06$ & \\
\hline
\end{tabular}




\begin{tabular}{|c|c|c|c|c|c|c|}
\hline$A G I^{2}$ & Description & $\begin{array}{c}\log 2 F C \\
\text { LOB-GR } \\
\text { CD/C }\end{array}$ & $\begin{array}{c}\text { p-value } \\
\text { LOB-GR } \\
\text { CD/C }\end{array}$ & $\begin{array}{c}\log 2 \mathrm{FC} \\
\text { LOB-GR } \\
\mathrm{D} / \mathrm{M}^{3}\end{array}$ & \begin{tabular}{|c|} 
p-value \\
LOB-GR \\
D/M
\end{tabular} & $\begin{array}{l}\text { Multiple } \\
\text { Binding } \\
\text { sites }^{5}\end{array}$ \\
\hline AT5G65140 & $\begin{array}{l}\text { trehalose-6-phosphate phosphatase, putative, similar to trehalose-6- } \\
\text { phosphate phosphatase (AtTPPB) (Arabidopsis thaliana) GI:2944180; } \\
\text { contains Pfam profile PF02358: Trehalose-phosphatase }\end{array}$ & 4.843 & $2.2 \mathrm{E}-16$ & 1.301 & $2.5 \mathrm{E}-06$ & \\
\hline AT1G55610 & $\begin{array}{l}\text { protein kinase family protein, contains Prosite:PS00107: Protein } \\
\text { kinases ATP-binding region signature }\end{array}$ & 2.284 & $2.5 \mathrm{E}-13$ & 1.286 & $2.9 \mathrm{E}-08$ & \\
\hline AT5G56320 & $\begin{array}{l}\text { expansin, putative (EXP14), similar to alpha-expansin } 3 \mathrm{Gl}: 6942322 \\
\text { from (Triphysaria versicolor); alpha-expansin gene family, } \\
\text { PMID:11641069 }\end{array}$ & 1.931 & 4.5E-06 & 1.284 & $1.2 \mathrm{E}-03$ & yes \\
\hline AT4G00080 & $\begin{array}{l}\text { invertase/pectin methylesterase inhibitor family protein, low similarity to } \\
\text { pectinesterase from Lycopersicon esculentum SP:Q43143, Arabidopsis } \\
\text { thaliana SP:Q42534; contains Pfam profile PF04043: Plant } \\
\text { invertase/pectin methylesterase inhibitor }\end{array}$ & 3.323 & 1.4E-09 & 1.283 & 1.6E-03 & \\
\hline AT5G43150 & expressed protein & 1.449 & $1.0 \mathrm{E}-06$ & 1.265 & $1.4 \mathrm{E}-05$ & \\
\hline AT4G31820 & $\begin{array}{l}\text { phototropic-responsive NPH3 family protein, contains NPH3 family } \\
\text { domain, Pfam:PF03000 (NPY1) }\end{array}$ & 2.581 & 1.1E-10 & 1.251 & $2.2 \mathrm{E}-05$ & yes \\
\hline AT2G34080 & $\begin{array}{l}\text { cysteine proteinase, putative, contains similarity to cysteine protease } \\
\text { SPCP1 GI:13491750 from (Ipomoea batatas) }\end{array}$ & 1.747 & $7.8 \mathrm{E}-07$ & 1.242 & $1.5 \mathrm{E}-04$ & \\
\hline AT5G53900 & expressed protein, similar to unknown protein (gb:AAF34833.1) & 1.663 & $2.8 \mathrm{E}-09$ & 1.239 & $7.2 \mathrm{E}-07$ & \\
\hline AT4G28560 & $\begin{array}{l}\text { leucine-rich repeat family protein (fragment), contains leucine rich- } \\
\text { repeat domains Pfam:PF00560, INTERPRO:IPR001611; }\end{array}$ & 2.837 & $1.6 \mathrm{E}-09$ & 1.235 & $5.0 \mathrm{E}-04$ & yes \\
\hline AT1G10650 & expressed protein & 3.493 & $3.9 \mathrm{E}-12$ & 1.212 & $1.4 \mathrm{E}-04$ & \\
\hline AT5G10130 & $\begin{array}{l}\text { pollen Ole e } 1 \text { allergen and extensin family protein, contains similarity to } \\
\text { pollen specific protein C13 precursor (Zea mays) SWISS-PROT:P33050 }\end{array}$ & 2.766 & $1.8 \mathrm{E}-10$ & 1.178 & $1.6 \mathrm{E}-04$ & yes \\
\hline AT4G27900 & expressed protein & 1.194 & $1.1 \mathrm{E}-06$ & 1.177 & $3.0 \mathrm{E}-06$ & \\
\hline AT2G24550 & $\begin{array}{l}\text { expressed protein, weak similarity to MTD1 (Medicago truncatula) } \\
\text { GI:9294810 }\end{array}$ & 2.962 & 4.7E-14 & 1.153 & 1.3E-06 & \\
\hline AT5G04770 & $\begin{array}{l}\text { amino acid permease family protein, similar to cationic amino acid } \\
\text { transporter-1 (Rattus norvegicus) GI:1589917; contains Pfam profile } \\
\text { PF00324: Amino acid permease }\end{array}$ & 1.912 & $1.6 \mathrm{E}-07$ & 1.138 & $3.6 \mathrm{E}-04$ & yes \\
\hline AT1G15670 & $\begin{array}{l}\text { kelch repeat-containing F-box family protein, similar to SP:Q9ER30 } \\
\text { Kelch-related protein } 1 \text { (Sarcosin) \{Rattus norvegicus\}; contains Pfam } \\
\text { profiles PF01344: Kelch motif, PF00646: F-box domain }\end{array}$ & 1.728 & $1.2 \mathrm{E}-09$ & 1.114 & 3.0E-06 & yes \\
\hline AT5G53890 & leucine-rich repeat transmembrane protein kinase, putative & 1.381 & 9.4E-09 & 1.093 & 9.0E-07 & \\
\hline AT2G24240 & $\begin{array}{l}\text { potassium channel tetramerisation domain-containing protein, contains } \\
\text { Pfam profile PF02214: } \mathrm{K}+\text { channel tetramerisation domain }\end{array}$ & 1.397 & $1.2 \mathrm{E}-07$ & 1.088 & $9.8 \mathrm{E}-06$ & \\
\hline AT3G07410 & $\begin{array}{l}\text { Ras-related GTP-binding family protein, contains Pfam profile: PF00071 } \\
\text { Ras family }\end{array}$ & 1.574 & 1.6E-09 & 1.060 & $2.0 \mathrm{E}-06$ & \\
\hline AT4G19810 & $\begin{array}{l}\text { glycosyl hydrolase family } 18 \text { protein, similar to chitinase/lysozyme } \\
\text { Gl:467689 from (Nicotiana tabacum) }\end{array}$ & 1.377 & $3.2 \mathrm{E}-08$ & 1.050 & 4.3E-06 & \\
\hline AT3G15357 & expressed protein & 1.122 & 7.0E-06 & 1.031 & $4.1 \mathrm{E}-05$ & \\
\hline AT3G58790 & $\begin{array}{l}\text { glycosyl transferase family } 8 \text { protein, contains Pfam profile: PF01501 } \\
\text { glycosyl transferase family 8; general stress protein gspA, Bacillus } \\
\text { subtilis, PIR:S16423 }\end{array}$ & 1.139 & 4.9E-07 & 1.016 & 5.3E-06 & \\
\hline AT1G26290 & expressed protein & 2.859 & $6.5 \mathrm{E}-10$ & 1.014 & 2.4E-03 & \\
\hline AT1G29440 & $\begin{array}{l}\text { auxin-responsive family protein, similar to auxin-induced protein 6B } \\
\text { (SP:P33083) (Glycine max) }\end{array}$ & -1.740 & 5.7E-06 & -1.008 & $5.1 \mathrm{E}-03$ & \\
\hline AT4G11290 & $\begin{array}{l}\text { peroxidase, putative, identical to peroxidase ATP19a (Arabidopsis } \\
\text { thaliana) gi:1546692:emb:CAA67337 }\end{array}$ & -1.016 & 7.6E-04 & -1.008 & $1.8 \mathrm{E}-03$ & \\
\hline AT3G43430 & $\begin{array}{l}\text { zinc finger (C3HC4-type RING finger) family protein, contains Pfam } \\
\text { profile: PF00097 zinc finger, C3HC4 type (RING finger) }\end{array}$ & -1.017 & 9.3E-06 & -1.021 & $1.8 \mathrm{E}-05$ & \\
\hline AT3G15650 & $\begin{array}{l}\text { phospholipase/carboxylesterase family protein, low similarity to } \\
\text { lysophospholipase I (Mus musculus) GI:1864159; contains Pfam profile } \\
\text { PF02230: Phospholipase/Carboxylesterase family }\end{array}$ & -1.183 & 7.0E-05 & -1.041 & $6.1 \mathrm{E}-04$ & \\
\hline AT2G25000 & $\begin{array}{l}\text { WRKY family transcription factor, contains Pfam profile: PF03106 } \\
\text { WRKY DNA -binding domain }\end{array}$ & -1.868 & $3.6 \mathrm{E}-10$ & -1.051 & 7.6E-06 & \\
\hline AT1G25230 & $\begin{array}{l}\text { purple acid phosphatase family protein, contains Pfam profile: PF00149 } \\
\text { calcineurin-like phosphoesterase; similar to purple acid phosphatase } \\
\text { (Gl:20257479) (Arabidopsis thaliana) }\end{array}$ & -1.154 & $6.1 \mathrm{E}-05$ & -1.055 & $3.6 \mathrm{E}-04$ & \\
\hline AT2G37640 & $\begin{array}{l}\text { expansin, putative (EXP3), identical to Alpha-expansin } 3 \text { precursor (At- } \\
\text { EXP3)(Arabidopsis thaliana) SWISS-PROT:O80932; alpha-expansin } \\
\text { gene family, PMID:11641069 }\end{array}$ & -1.541 & $2.8 \mathrm{E}-05$ & -1.067 & $3.1 \mathrm{E}-03$ & yes \\
\hline AT3G49220 & $\begin{array}{l}\text { pectinesterase family protein, contains Pfam profile: PF01095 } \\
\text { pectinesterase }\end{array}$ & -1.205 & $1.2 \mathrm{E}-07$ & -1.082 & $1.4 \mathrm{E}-06$ & yes \\
\hline
\end{tabular}




\begin{tabular}{|c|c|c|c|c|c|c|}
\hline $\mathbf{A G I ^ { 2 }}$ & Description & $\begin{array}{c}\text { log2FC } \\
\text { LOB-GR } \\
\text { CD/C } \text { C }^{3}\end{array}$ & $\begin{array}{c}\text { p-value } \\
\text { LOB-GR } \\
\text { CD/C }\end{array}$ & $\begin{array}{l}\text { log2FC } \\
\text { LOB-GR } \\
D / M^{3}\end{array}$ & $\begin{array}{c}\text { p-value } \\
\text { LOB-GR } \\
\text { D/M }\end{array}$ & $\begin{array}{c}\text { Multiple } \\
\text { Binding } \\
\text { sites }^{5}\end{array}$ \\
\hline AT1G19450 & $\begin{array}{l}\text { integral membrane protein, putative / sugar transporter family protein, } \\
\text { similar to GB:U43629 Gl: } 1209756 \text { integral membrane protein from (Beta } \\
\text { vulgaris); contains Pfam profile PF00083: major facilitator superfamily } \\
\text { protein; contains TIGRfam TIGR00879: Suga }\end{array}$ & -1.408 & $4.9 \mathrm{E}-09$ & -1.088 & 7.2E-07 & \\
\hline AT1G64640 & $\begin{array}{l}\text { plastocyanin-like domain-containing protein, contains } \\
\text { InterPro:IPR003245 plastocyanin-like domain }\end{array}$ & -1.482 & $1.8 \mathrm{E}-05$ & -1.093 & $1.2 \mathrm{E}-03$ & \\
\hline AT5G20740 & $\begin{array}{l}\text { invertase/pectin methylesterase inhibitor family protein, low similarity to } \\
\text { pectinesterase from Arabidopsis thaliana SP:Q42534, Lycopersicon } \\
\text { esculentum SP:Q43143; contains Pfam profile PF04043: Plant } \\
\text { invertase/pectin methylesterase inhibitor }\end{array}$ & -1.068 & $1.2 \mathrm{E}-04$ & -1.102 & $1.7 \mathrm{E}-04$ & \\
\hline AT3G57010 & $\begin{array}{l}\text { strictosidine synthase family protein, similar to strictosidine synthase } \\
\text { (Rauvolfia serpentina)(SP:P15324) }\end{array}$ & 1.602 & $2.5 \mathrm{E}-06$ & -1.104 & $5.4 \mathrm{E}-04$ & \\
\hline AT5G64330 & $\begin{array}{l}\text { non-phototropic hypocotyl } 3 \text { (NPH3), identical to non-phototropic } \\
\text { hypocotyl } 3 \text { (Arabidopsis thaliana) gi:6224712:gb:AAF05914, } \\
\text { PMID:10542152 }\end{array}$ & -1.561 & 7.0E-11 & -1.138 & $6.2 \mathrm{E}-08$ & ye \\
\hline AT2G06925 & $\begin{array}{l}\text { phospholipase A2 family protein, similar to secretory low molecular } \\
\text { weight phospholipase A2 beta (Arabidopsis thaliana) GI:25992715; } \\
\text { contains INTERPRO domain IPR001211 phospholipase A2 }\end{array}$ & -1.171 & $9.4 \mathrm{E}-08$ & -1.166 & 2.6E-07 & \\
\hline AT5G62920 & $\begin{array}{l}\text { two-component responsive regulator / response regulator } 6 \text { (ARR6), } \\
\text { identical to response regulator } 6 \text { (Arabidopsis thaliana) Gl:3953601 }\end{array}$ & -1.078 & $5.9 \mathrm{E}-06$ & -1.168 & $4.2 \mathrm{E}-06$ & ye \\
\hline AT1G62480 & $\begin{array}{l}\text { vacuolar calcium-binding protein-related, contains weak similarity to } \\
\text { vacuolar calcium binding protein (Raphanus sativus) } \\
\text { gi:9049359:dbj:BAA99394 }\end{array}$ & -1.646 & $6.2 \mathrm{E}-08$ & -1.168 & $1.9 \mathrm{E}-05$ & \\
\hline AT1G58360 & $\begin{array}{l}\text { amino acid permease I (AAP1), identical to a } \\
\text { GI:22641 from (Arabidopsis thaliana) }\end{array}$ & -1.398 & $3.4 \mathrm{E}-05$ & -1.179 & $5.0 \mathrm{E}-04$ & \\
\hline AT5G53550 & $\begin{array}{l}\text { transporter, putative, similar to iron-phytosiderop } \\
\text { yellow stripe } 1 \text { (Zea mays) Gl: } 10770865 \text {; contain } \\
\text { PF03169: OPT oligopeptide transporter protein }\end{array}$ & .119 & 7E-07 & .182 & $6 \mathrm{E}-07$ & \\
\hline AT3G44990 & $\begin{array}{l}\text { I xyloglucan } \\
\text { Ican transferase, putative }\end{array}$ & .683 & $6 \mathrm{E}-07$ & 1.184 & $6.8 \mathrm{E}-05$ & \\
\hline AT1G04240 & $\begin{array}{l}\text { auxin-responsive protein / indoleacetic acid-induced protein } 3 \text { (IAA3), } \\
\text { identical to SP:Q38822 Auxin-responsive protein IAA3 (Indoleacetic acid- } \\
\text { induced protein 3) \{Arabidopsis thaliana\}; EST gb:T04296 comes from } \\
\text { this gene }\end{array}$ & -1.134 & 3.1E-07 & -1.189 & 3.5E-07 & \\
\hline AT2G31750 & $\begin{array}{l}\text { UDP-glucoronosyl/UDP-glucosyl transferase family protein, } \\
\text { Pfam profile: PF00201 UDP-glucoronosyl and UDP-glucosy }\end{array}$ & 1.056 & 2E-07 & .212 & $0 \mathrm{E}-07$ & \\
\hline AT1G71880 & $\begin{array}{l}\text { sucrose transp } \\
\text { sucrose-proton }\end{array}$ & -2.483 & $4.2 \mathrm{E}-12$ & -1.214 & $1.8 \mathrm{E}-06$ & ye \\
\hline AT2G28790 & $\begin{array}{l}\text { osmotin-like protein, putative, similar } \\
\text { protein precursor }\{\text { Lycopersicon escul } \\
\text { PF00314: Thaumatin family }\end{array}$ & -1.131 & $4.9 \mathrm{E}-06$ & 1.237 & 3.0E-06 & \\
\hline AT5G10430 & $\begin{array}{l}\text { arabinogalactan-protein (AGP4), identical to } \\
\text { gi_3883126_gb_AAC77826 }\end{array}$ & -1.364 & $1.2 \mathrm{E}-04$ & -1.272 & $5.2 \mathrm{E}-04$ & \\
\hline AT3G23730 & $\begin{array}{l}\text { xyloglucan:xyloglucosyl transferase, putative / xyloglucan } \\
\text { endotransglycosylase, putative / endo-xyloglucan transferase, putative, } \\
\text { similar to xyloglucan endotransglycosylase-related protein GI:1244760 } \\
\text { from (Arabidopsis thaliana) }\end{array}$ & -1.205 & 4.0E-07 & -1.289 & $3.4 \mathrm{E}-07$ & \\
\hline AT4G19120 & $\begin{array}{l}\text { early-responsive to dehydration stress protein (ERD3), identical to } \\
\text { ERD3 protein (Arabidopsis thaliana) Gl:15320410; contains Pfam profile } \\
\text { PF03141: Putative methyltransferase; identical to cDNA ERD3 } \\
\text { Gl:15320409 }\end{array}$ & -1.048 & 7.0E-06 & -1.295 & 7.3E-07 & \\
\hline AT2G1 & $\begin{array}{l}\text { gibberellin-regulate } \\
\text { regulated protein } 4 \\
\text { Pfam profile PF027 }\end{array}$ & -1.361 & 8.1E-07 & -1.298 & $3.5 \mathrm{E}-06$ & \\
\hline AT3G47510 & expressed protein & $\mid-1.247$ & $1.8 \mathrm{E}-06$ & -1.309 & $2.0 \mathrm{E}-06$ & \\
\hline AT5G63180 & protein, similar to pectate lyase GP:14289169 from & -1.561 & $6.7 \mathrm{E}-07$ & -1.309 & $1.6 \mathrm{E}-05$ & \\
\hline AT3G10720 & $\begin{array}{l}\text { pectinesterase, putative, contains similarity to pectinesterase from Vitis } \\
\text { vinifera Gl:15081598, Prunus persica SP:Q43062; contains Pfam profile } \\
\text { PF01095 pectinesterase }\end{array}$ & -1.023 & 7.0E-06 & -1.311 & 4.5E-07 & ye \\
\hline AT4G03190 & $\begin{array}{l}\text { F-box family protein (FBL18), almost identical to GRR1-like protein } 1 \\
\text { GI:12658970 from (Arabidopsis thaliana); similar to leucine-rich repeats } \\
\text { containing F-box protein FBL3 (Gl:5919219) (Homo sapiens); similar to } \\
\text { F-box protein FBL2 (GI:6063090) (Homo sap }\end{array}$ & -1.502 & 2.2E-06 & -1.317 & $2.7 \mathrm{E}-05$ & \\
\hline 1G13710 & $\begin{array}{l}\text { cytochrome P450 family protein, similar to cytochrome P450 78A1 } \\
\text { (SP:P48420) Gl:349717 from (Zea mays) }\end{array}$ & -1.058 & $1.1 \mathrm{E}-04$ & -1.338 & $1.2 \mathrm{E}-05$ & \\
\hline
\end{tabular}




\begin{tabular}{|c|c|c|c|c|c|c|}
\hline $\mathbf{A G I ^ { 2 }}$ & Description & $\mid \begin{array}{c}\log 2 \mathrm{FC} \\
\mathrm{LOB}-\mathrm{GR} \\
\mathrm{CD} / \mathrm{C}^{3}\end{array}$ & \begin{tabular}{|c|}
-value \\
LOB-GR \\
CD/C
\end{tabular} & $\begin{array}{c}\text { log2FC } \\
\text { LOB-GR } \\
D / M^{3}\end{array}$ & \begin{tabular}{|c|} 
p-value \\
LOB-GR \\
D/M
\end{tabular} & $\begin{array}{c}\text { Multiple } \\
\text { Binding } \\
\text { sites }^{5} \\
\end{array}$ \\
\hline AT1G67810 & $\begin{array}{l}\text { Fe-S metabolism associated domain-containing protein, contains Pfam } \\
\text { PF02657: Fe-S metabolism associated domain }\end{array}$ & -1.060 & $1.8 \mathrm{E}-05$ & -1.363 & $1.1 \mathrm{E}-06$ & \\
\hline AT3G02170 & expressed protein & -1.181 & $9.3 \mathrm{E}-05$ & -1.365 & $3.2 \mathrm{E}-05$ & \\
\hline AT5G23010 & $\begin{array}{l}\text { 2-isopropylmalate synthase } 3 \text { (IMS3), identical to 2-isopropylmalate } \\
\text { synthase (IMS3) (Arabidopsis thaliana) Gl:15983745; identical to cDNA } \\
\text { 2-isopropylmalate synthase (IMS3) Gl:15983744 }\end{array}$ & -1.632 & 4.1E-07 & -1.370 & $1.0 \mathrm{E}-05$ & \\
\hline AT1G11545 & $\begin{array}{l}\text { xyloglucan:xyloglucosyl transferase, putative / xyloglucan } \\
\text { endotransglycosylase, putative / endo-xyloglucan transferase, putative, } \\
\text { similar to endo-xyloglucan transferase GI:2244732 from (Gossypium } \\
\text { hirsutum) }\end{array}$ & -1.692 & $1.8 \mathrm{E}-09$ & -1.373 & 1.7E-07 & \\
\hline AT2G31730 & $\begin{array}{l}\text { ethylene-responsive protein, putative, similar to ethylene-inducible } \\
\text { ER33 protein (Lycopersicon esculentum) gi:5669656:gb:AAD46413 }\end{array}$ & -1.275 & 2.1E-07 & -1.414 & $1.3 \mathrm{E}-07$ & \\
\hline AT1G20190 & $\begin{array}{l}\text { expansin, putative (EXP11), similar to GB:U30460 from (Cucumis } \\
\text { sativus); alpha-expansin gene family, PMID:11641069 }\end{array}$ & -1.262 & $5.1 \mathrm{E}-03$ & -1.467 & $3.4 \mathrm{E}-03$ & ye \\
\hline AT5G45670 & $\begin{array}{l}\text { GDSL-motif lipase/hydrolase family protein, similar to family II lipases } \\
\text { EXL3 Gl:15054386, EXL1 Gl:15054382, EXL2 Gl:15054384 from } \\
\text { (Arabidopsis thaliana); contains Pfam profile PF00657: GDSL-like } \\
\text { Lipase/Acylhydrolase }\end{array}$ & -1.277 & $6.4 \mathrm{E}-09$ & -1.468 & 2.6E-09 & \\
\hline AT1G29500 & $\begin{array}{l}\text { auxin-responsive protein, putative, similar to auxin-induced protein 6B } \\
\text { (SP:P33083) (Glycine max) }\end{array}$ & -1.265 & 8.7E-07 & -1.478 & 2.1E-07 & \\
\hline AT2G17740 & DC1 domain-containing protein & -1.613 & $8 \mathrm{E}-06$ & -1.489 & $2.8 \mathrm{E}-05$ & \\
\hline AT1G52190 & $\begin{array}{l}\text { proton-dependent oligopeptide tran } \\
\text { Pfam profile: PF00854 POT family }\end{array}$ & .675 & -06 & -1.491 & -05 & \\
\hline AT2G21220 & $\begin{array}{l}\text { auxin-responsive protein, putative, similar to auxin-induced protein } \\
\text { TGSAUR22 (Gl:10185820) (Tulipa gesneriana) }\end{array}$ & .634 & $6 \mathrm{E}-09$ & 1.492 & $9 \mathrm{E}-08$ & \\
\hline AT4G23810 & $\begin{array}{l}\text { WRKY family transcription factor, AR411 - Arabidopsis thaliana (thale } \\
\text { cress), PID:g1669603 }\end{array}$ & 1.761 & 1E-06 & .531 & 3E-05 & ye \\
\hline AT4G36670 & $\begin{array}{l}\text { mannitol transporter, putative, similar to mannitol transporter (Apium } \\
\text { graveolens var. dulce) Gl:12004316; contains Pfam profile PF00083: } \\
\text { major facilitator superfamily protein }\end{array}$ & -1.144 & $6.6 \mathrm{E}-06$ & -1.551 & 2.0E-07 & yes \\
\hline AT1G2 & $\begin{array}{l}\text { [AT1G29430, auxin-responsive family protein, similar to auxin-induced } \\
\text { protein 10a 6B \{SP:P33083) (Glycine max) ]; [AT5G27780, auxin- } \\
\text { responsive family protein, similar to Auxin-induced protein 10A5 } \\
\text {;(SP:P33079) (Glycine max) ] }\end{array}$ & -2.139 & 1E-08 & .556 & $8 \mathrm{E}-06$ & \\
\hline AT4G00050 & $\begin{array}{l}\text { basic helix-loop-helix (bHLH) family protein, contains Pfam profile: } \\
\text { PF00010 helix-loop-helix DNA-binding domain }\end{array}$ & -1.315 & $7.5 \mathrm{E}-06$ & -1.567 & $1.4 \mathrm{E}-06$ & . \\
\hline AT1G29510 & $\begin{array}{l}\text { auxin-responsive protein, putative, similar to auxin-induced protein 6B } \\
\text { (SP:P33083) (Glycine max) }\end{array}$ & -1.469 & $2 \mathrm{E}-06$ & -1.586 & 8.7E-07 & \\
\hline AT5G61660 & glycine-rich protein & .335 & $0 \mathrm{E}-07$ & 1.598 & $.5 \mathrm{E}-07$ & \\
\hline AT3G07010 & $\begin{array}{l}\text { pectate lyase family protein, similar to pectate lyase GP:14531296 from } \\
\text { (Fragaria x ananassa) }\end{array}$ & .382 & $6 \mathrm{E}-06$ & 1.639 & $5 \mathrm{E}-06$ & \\
\hline AT5G46050 & $\begin{array}{l}\text { proton-dependent oligopeptide transport (POT) family protein, contains } \\
\text { Pfam profile: PF00854 POT family }\end{array}$ & -1.190 & 4.1E-05 & -1.681 & 7.8E-07 & \\
\hline AT1G62500 & $\begin{array}{l}\text { protease inhibitor/seed storage/lipid transfer protein (LTP) family } \\
\text { protein, similar to auxin down regulated GB:X69640 Gl:296442 from } \\
\text { (Glycine max); contains Pfam profile PF00234: Protease inhibitor/seed } \\
\text { storage/LTP family }\end{array}$ & -1.781 & 3.6E-08 & -1.682 & $2.5 \mathrm{E}-07$ & \\
\hline AT1G78970 & $\begin{array}{l}\text { lupeol synthase (LUP1) / 2,3-oxidosqualene-triterpenoid cyclase, } \\
\text { identical to lupeol synthase GI:1762150 from (Arabidopsis thaliana), 2,3- } \\
\text { oxidosqualene-triterpenoid cyclase (Arabidopsis thaliana) GI:2738027; } \\
\text { contains Pfam profile PF00432: Prenyltransfera }\end{array}$ & -1.140 & $1.1 \mathrm{E}-03$ & -1.692 & $2.9 \mathrm{E}-05$ & \\
\hline AT3G50440 & $\begin{array}{l}\text { similar to esterase, putative [Arabidopsis thaliana] (TAIR:At2g23620.1); } \\
\text { similar to methyl jasmonate esterase [Solanum tuberosum] } \\
\text { (GB:AAV87151.1); contains InterPro domain } \\
\text { Esterase/lipase/thioesterase (InterPro:IPR000379); contains InterPro } \\
\text { domain Alpha/ }\end{array}$ & -1.123 & $1.1 \mathrm{E}-04$ & -1.751 & $6.9 \mathrm{E}-07$ & \\
\hline 250 & $\begin{array}{l}\text { auxin-responsive family protein, similar to auxin-induced protein } \\
\text { TGSAUR22 (GI:10185820) (Tulipa gesneriana and auxin-induced } \\
\text { protein 6B (SP:P33083) (PIR:T10942) (Glycine max) }\end{array}$ & 1.141 & 0E-03 & 1.812 & $0 \mathrm{E}-05$ & \\
\hline AT2G41090 & $\begin{array}{l}\text { calmodulin-like calcium-binding protein, } 22 \mathrm{kDa} \text { (CaBP-22), identical to } \\
\text { SP:P30187 } 22 \mathrm{kDa} \text { calmodulin-like calcium-binding protein (CABP-22) } \\
\text { (Arabidopsis thaliana) }\end{array}$ & -1.093 & $1.4 \mathrm{E}-05$ & -1.815 & 2.5E-08 & \\
\hline IG12 & $\begin{array}{l}\text { flavin-containing monooxygenase family protein / FMO family protein, } \\
\text { low similarity to FMO2 from Homo sapiens (SP:Q99518); contains Pfam } \\
\text { profile: PF00743 Flavin-binding monooxygenase-like }\end{array}$ & -1.385 & $8.2 \mathrm{E}-09$ & -1.862 & $4.1 \mathrm{E}-10$ & \\
\hline
\end{tabular}




\begin{tabular}{|c|c|c|c|c|c|c|}
\hline$A G I^{2}$ & Description & $\begin{array}{c}\log 2 F C \\
\text { LOB-GR } \\
\text { CD/C }\end{array}$ & $\begin{array}{l}\text { p-value } \\
\text { LOB-GR } \\
\text { CD/C }\end{array}$ & $\begin{array}{c}\log 2 \mathrm{FC} \\
\text { LOB-GR } \\
\mathrm{D} / \mathrm{M}^{3}\end{array}$ & $\begin{array}{c}\text { p-value } \\
\text { LOB-GR } \\
\text { D/M }\end{array}$ & $\begin{array}{l}\text { Multiple } \\
\text { Binding } \\
\text { sites }^{5}\end{array}$ \\
\hline AT2G39200 & $\begin{array}{l}\text { seven transmembrane MLO family protein / MLO-like protein 12 } \\
\text { (MLO12), identical to SP:O80961 MLO-like protein } 12 \text { (AtMlo12) } \\
\text { \{Arabidopsis thaliana\}, membrane protein Mlo12 (Arabidopsis thaliana) } \\
\text { gi:14091594:gb:AAK53805; similar to MLO protein SWISS-PROT:P9 }\end{array}$ & -1.547 & $2.0 \mathrm{E}-06$ & -1.874 & $2.9 \mathrm{E}-07$ & \\
\hline AT5G64570 & glycosyl hydrolase family 3 protein & -1.186 & 1.6E-05 & -1.889 & $5.9 \mathrm{E}-08$ & \\
\hline AT3G12710 & $\begin{array}{l}\text { methyladenine glycosylase family protein, similar to SP:P05100 DNA-3- } \\
\text { methyladenine glycosylase I (EC 3.2.2.20) (3-methyladenine-DNA } \\
\text { glycosylase I, constitutive) \{Escherichia coli\}; contains Pfam profile } \\
\text { PF03352: Methyladenine glycosylase }\end{array}$ & -1.009 & 7.1E-04 & -1.895 & 5.9E-07 & \\
\hline AT2G13610 & ABC transporter family protein & -1.061 & 4.1E-05 & -1.897 & $3.0 \mathrm{E}-08$ & \\
\hline & $\begin{array}{l}\text { protein phosphatase 2C family protein / PP2C family protein, similar to } \\
\text { Ser/Thr protein phosphatase 2C (PP2C6) (Gl:15020818) (Arabidopsis } \\
\text { thaliana); similar to protein phosphatase 2C (Gl:3608412) }\end{array}$ & & & & & \\
\hline AT5G02760 & (Mesembryanthemum crystallinum); contains Pfam PF00481: P & -1.505 & $6.9 \mathrm{E}-08$ & -1.899 & $6.0 \mathrm{E}-09$ & \\
\hline AT5G50335 & expressed protein & -1.996 & 4.1E-07 & -2.037 & $6.7 \mathrm{E}-07$ & \\
\hline AT2G40610 & $\begin{array}{l}\text { expansin, putative (EXP8), similar to expansin } 2 \text { Gl:7025493 from } \\
\text { (Zinnia elegans); alpha-expansin gene family, PMID:11641069 }\end{array}$ & -1.247 & 8.6E-03 & -2.061 & $1.8 \mathrm{E}-04$ & yes \\
\hline AT3G51860 & $\begin{array}{l}\text { cation exchanger, putative (CAX3), similar to high affinity calcium } \\
\text { antiporter CAX1 (Arabidopsis thaliana) gi:9256741:gb:AAB05913; } \\
\text { Ca2+:Cation Antiporter (CaCA) Family member PMID:11500563; non- } \\
\text { consensus AT-acceptor splice site at intron } 1\end{array}$ & -2.347 & $4.2 \mathrm{E}-09$ & -2.092 & $9.2 \mathrm{E}-08$ & \\
\hline AT4G34220 & $\begin{array}{l}\text { leucine-rich repeat transmembrane protein kinase, putative, protein } \\
\text { kinase TMKL1, Arabidopsis thaliana, PID:E353150 }\end{array}$ & -1.092 & $1.9 \mathrm{E}-05$ & -2.130 & 2.6E-09 & \\
\hline AT5G01210 & $\begin{array}{l}\text { transferase family protein, contains Pfam profile PF02458 transferase } \\
\text { family }\end{array}$ & -2.368 & $9.7 \mathrm{E}-12$ & -2.399 & $1.0 \mathrm{E}-10$ & yes \\
\hline AT5G62280 & expressed protein & -4.056 & $4.2 \mathrm{E}-12$ & -2.838 & 1.1E-08 & yes \\
\hline AT4G02330 & $\begin{array}{l}\text { pectinesterase family protein, contains Pfam profile: PF01095 } \\
\text { pectinesterase }\end{array}$ & -1.647 & 1.1E-05 & -2.960 & 5.3E-09 & s \\
\hline
\end{tabular}

